\title{
Bucket-handle tear of the triangular fibrocartilage complex: case report of a complex peripheral injury with separation of the distal radioulnar ligaments from the articular disc
}

\author{
Nicolas Theumann • Ehab M. Kamel • \\ Christof Bollmann • Michael Sturzenegger • Fabio Becce
}

Received: 26 December 2010 /Revised: 22 August 2011 /Accepted: 24 August 2011 /Published online: 16 September 2011

(C) ISS 2011

\begin{abstract}
Palmer previously proposed a classification system of triangular fibrocartilage complex (TFCC) injuries that proved to be useful in directing clinical management. However, dorsal peripheral tears (variants of class 1C) were not described and have rarely been reported in the literature since. We herewith present a rare case of bucket-handle tear of the TFCC. To our knowledge, this is the first case demonstrating partial separation of both the palmar and dorsal distal radioulnar ligaments (DRULs) from the articular disc. The particular wrist magnetic resonance (MR) arthrographic findings of this unusual complex peripheral TFCC tear (a variant of both class $1 \mathrm{~B}$ and 1C) were nicely appreciated upon sagittal reformatted images.
\end{abstract}

\section{Introduction}

Palmer previously proposed a classification system of triangular fibrocartilage complex (TFCC) injuries that has

Ehab M. Kamel and Fabio Becce contributed equally to this work.

N. Theumann $\cdot$ E. M. Kamel $\cdot$ F. Becce $(\bowtie)$

Department of Diagnostic and Interventional Radiology,

Centre Hospitalier Universitaire Vaudois, University of Lausanne,

Rue du Bugnon 46,

1011 Lausanne, Switzerland

e-mail: fabio.becce@chuv.ch

C. Bollmann

Department of Plastic and Hand Surgery,

Clinique Longeraie and Centre Hospitalier Universitaire Vaudois,

1003 Lausanne, Switzerland

\section{Sturzenegger}

Division of Plastic and Hand Surgery,

Clinique Longeraie and Centre Médical de Vidy,

1007 Lausanne, Switzerland been widely applied by radiologists and hand surgeons, and which proved to be useful in directing clinical management [1-3]. This classification differentiates traumatic (class 1) from degenerative (class 2) injuries. Traumatic lesions are further categorized as central (class 1A) or peripheral (classes 1B-1D). Distal tears (class 1C) imply injury to the palmar ulnocarpal (ulnolunate and/or ulnotriquetral) ligaments. However, dorsal peripheral tears, with or without ulnocarpal ligaments injury, were not described in Palmer classification system and have rarely been reported in the literature since [4-6]. Those kind of peripheral tears are important to recognize, as they may lead to distal radioulnar joint (DRUJ) and/or wrist instability [7, 8]. Furthermore, those injuries portend good healing after open or arthroscopic surgical repair $[4,9]$.

We herewith present a rare case of bucket-handle tear of the TFCC. To our knowledge, this is the first case demonstrating partial separation of both the palmar and dorsal distal radioulnar ligaments (DRULs) from the articular disc. The particular wrist magnetic resonance (MR) arthrographic findings of this unusual complex peripheral TFCC tear (a variant of both class 1B and 1C) were nicely appreciated upon sagittal reformatted images.

\section{Case report}

A 44-year-old manual worker visited our hospital with acute onset of ulnar-sided wrist pain after twisting his right wrist. His past medical history was unremarkable. On physical examination, he presented diffuse tenderness with pain on deep palpation at the ulnar aspect of the wrist. Posteroanterior and lateral wrist radiographs (not shown) demonstrated no fracture. The ulnar variance was negative and Gilula's arcs were not disrupted. A diagnosis of moderate (grade 2) wrist sprain was 
made and the patient treated with non-steroidal antiinflammatory drugs and splint immobilization during 4 weeks.

Three months later, the patient could not return to work and was dismissed. A standard wrist MR imaging (not shown) was performed in another institution on a 1.5-T unit and interpreted as normal.

Six months later, he still suffered from ulnar-sided wrist pain and reduced grip strength in activities of daily living. The ulnar fovea sign was positive and pain could be reproduced by the ulnocarpal stress test (both are sensitive screening tests for an intra-articular disorder $[10,11])$. The piano-key test (a provocative maneuver assessing DRUJ stability $[10,11])$ was also positive. Accordingly, a threecompartment MR arthrography of the wrist was performed. Arthrography was carried out under fluoroscopic guidance, with a dorsal approach. First, the midcarpal joint was punctured at the triquetrohamate space and a few milliliters of a mixture of $9 \mathrm{ml}$ of iodinated contrast material (Accupaque 300, GE Healthcare), $0.05 \mathrm{ml}$ of gadoteric acid (Dotarem, Guerbet), $1 \mathrm{ml}$ of lidocaine hydrochloride (Rapidocain 1\%, Sintetica) and one drop of epinephrine hydrochloride (Adrenalin, Sintetica) were injected. As a
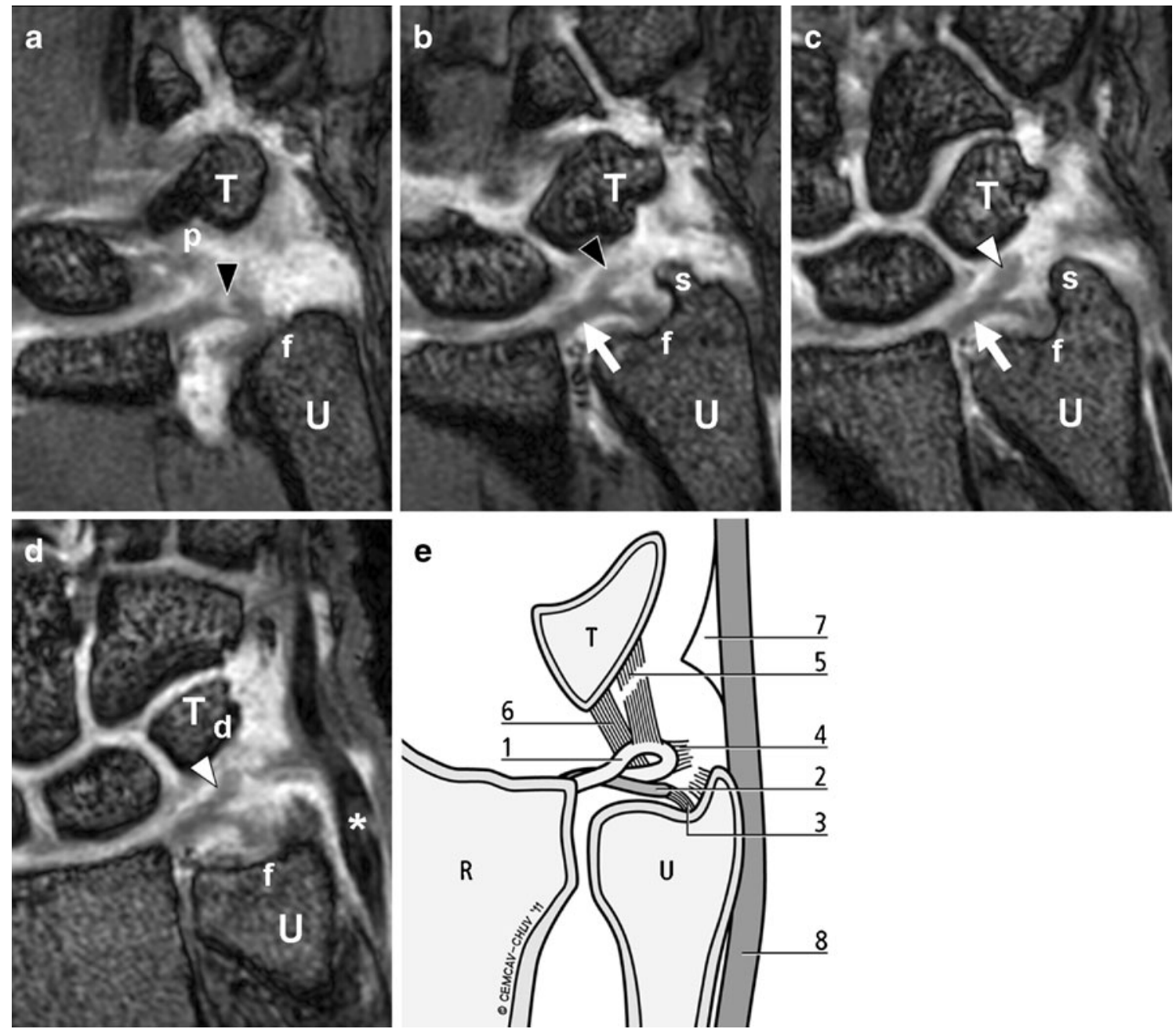

Fig. 1 a-d Left to right: sequential (palmar to dorsal) coronal T1weighted (repetition time/echo time, $11.2 \mathrm{~ms} / 3.5 \mathrm{~ms}$ ) custom volumetric interpolated breathhold examination (VIBE) MR arthrographic images of the right wrist display partial separation of the palmar (black arrowhead) and dorsal (white arrowhead) distal radioulnar ligaments (DRULs) from the articular disc (arrow). The dorsal ulnotriquetral ligament $(d)$ and the distal lamina of triangular fibrocartilage complex (TFCC) attachment on the ulnar styloid process (s) are torn. On the contrary, the articular disc, the palmar ulnotriquetral ligament $(p)$ and the proximal lamina of TFCC attachment on the ulnar fovea $(f)$ are preserved. This complex peripheral lesion represents a bucket-handle tear of the TFCC, with

the distal lamina of the TFCC and the DRULs partially subluxated distal to the articular disc. The custom VIBE MR sequence was acquired in free breathing keeping the same contrast features but after removing all interpolations in slice and phase direction. $T=$ triquetrum, $U=$ ulna, $*=$ extensor carpi ulnaris (ECU) tendon. e Illustrative diagram depicting the aforementioned lesion of the triangular fibrocartilage complex (TFCC): 1, palmar and dorsal distal radioulnar ligaments (DRULs); 2, articular disc; 3, insertion of TFCC proximal lamina on the ulnar fovea; 4 , insertion of TFCC distal lamina on the ulnar styloid process; 5, dorsal ulnotriquetral ligament; 6, palmar ulnotriquetral ligament; 7, meniscus homologue; 8 , extensor carpi ulnaris (ECU) tendon 
communication with the radiocarpal joint was noted through a central tear of the lunotriquetral ligament, approximately $6 \mathrm{ml}$ of the mixture was injected in order to fully distend the midcarpal and radiocarpal joints. No communication with the DRUJ was observed, even after active wrist movements with spot filming. Consequently, the DRUJ was punctured at the radial aspect of the ulnar head and about $2 \mathrm{ml}$ of the mixture were injected. MR imaging was then performed on a 3-T unit (Trio, Siemens, Erlangen, Germany) with a dedicated wrist coil (Alpha III Tx/Rx Quadrature, Siemens). Upon coronal isotropic high-resolution (section thickness $0.4 \mathrm{~mm}$ ) T1-weighted (repetition time/echo time, $11.2 \mathrm{~ms} / 3.5 \mathrm{~ms}$ ) custom volumetric interpolated breathhold examination (VIBE)
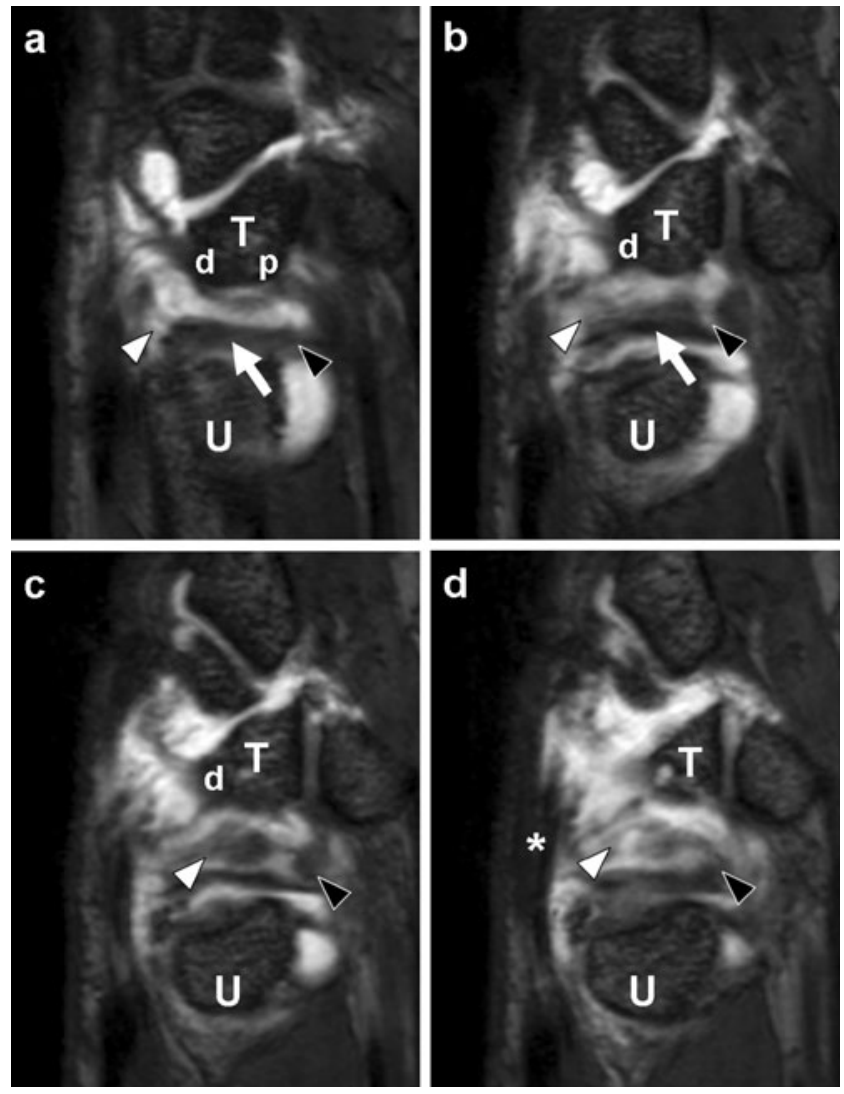

Fig. 2 a-d Left to right: sequential (radial to ulnar) sagittal reformatted T1-weighted (repetition time/echo time, $11.2 \mathrm{~ms} / 3.5 \mathrm{~ms}$ ) custom volumetric interpolated breathhold examination (VIBE) MR arthrographic images of the right wrist show partial separation of the palmar (black arrowhead) and dorsal (white arrowhead) distal radioulnar ligaments (DRULs) from the articular disc (arrow). The dorsal ulnotriquetral ligament $(d)$ is torn, but the palmar ulnotriquetral ligament $(p)$ is intact. This bucket-handle tear of the triangular fibrocartilage complex (TFCC) mimics the appearance of a pair of sunglasses (c), with the dorsal DRUL partially floating within the intra-articular contrast material, between the preserved articular disc and the triquetrum $(T)$. The custom VIBE MR sequence was acquired in free breathing keeping the same contrast features but after removing all interpolations in slice and phase direction. $U=\mathrm{ulna},{ }^{*}=$ extensor carpi ulnaris (ECU) tendon (acquired in free breathing without changing contrast features but after removing all interpolations in slice and phase direction) MR arthrographic images (Fig. 1a-d), a complex peripheral TFCC tear was noted. Indeed, the dorsal ulnotriquetral ligament and the distal lamina of TFCC attachment on the ulnar styloid process were torn (Figs. 1c-d and 2a-c). Moreover, the palmar and dorsal DRULs were partially separated from the articular disc and subluxated distal to it (Figs. $1 b-d$ and $2 c-d$ ). Interestingly, no communication between the radiocarpal joint and the DRUJ could be noted at the time of arthrography, as the articular disc and the proximal lamina of TFCC attachment on the ulnar fovea were preserved (Fig. 1a-d). Besides, the extensor carpi ulnaris (ECU) tendon and its tendon sheath were both intact. Upon a sagittal reformatted (section thickness $0.4 \mathrm{~mm}$ ) T1-weighted VIBE MR arthrographic view at the level of the TFCC (Fig. 2c), this complex peripheral TFCC tear mimicked the appearance of a pair of sunglasses.

The bucket-handle tear of the TFCC was subsequently confirmed at arthroscopy of the radiocarpal joint through the 3-4 portal (Fig. 3). The articular disc was intact and no communication was seen between the radiocarpal joint and the DRUJ (Fig. 3a). A complex peripheral tear partially separated the DRULs from the articular disc (Fig. 3b). However, the palmar and dorsal ulnocarpal ligaments could not be assessed at arthroscopy. This tear was then repaired using a mini-open suture anchor technique, with reinsertion of the distal lamina of the TFCC on the ulnar styloid process. Unfortunately, no clinical improvement was observed in the few days following surgery. The patient was then lost to follow-up at 1 month when he left the country for personal reasons.
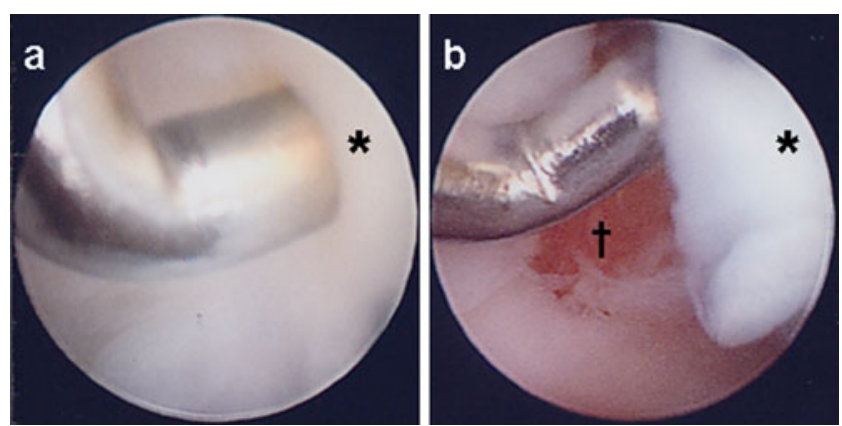

Fig. 3 Arthroscopic images of the radiocarpal joint. Distal is to the left and dorsal is to the top of the pictures. The arthroscope is inserted dorsally through the 3-4 portal, while the instruments are passed between the extensor tendons using the $6 \mathrm{R}$ portal. a The articular disc $(*)$ is intact and no communication between the radiocarpal joint and distal radioulnar joint (DRUJ) is seen. b The hook probe is passed through the complex peripheral tear that partially separates the distal radioulnar ligaments (DRULs) from the articular disc $\left(^{*}\right) . \dagger=$ prestyloid recess 


\section{Discussion}

Ulnar-sided wrist pain is a diagnostic challenge for both hand surgeons and radiologists due to the small and intricate anatomical structures involved. The differential diagnosis is wide, with tears of the TFCC representing one of the most common causes [10, 12]. History and physical examination may help to differentiate tears of the articular disc from lesions of the peripheral portion of the TFCC [10-13]. Indeed, patients with articular disc injuries will complain of discomfort during pro/supination of the wrist, whereas peripheral destabilizing TFCC tears will induce a more constant pain in activities of daily living and a greater limitation of range of motion [11, 12]. Moreover, the ulnar fovea sign was reported to be a sensitive (95.2\%) and specific $(86.5 \%)$ clinical maneuver for foveal disruptions of the DRULs and/or ulnotriquetral ligament injuries [13]. However, as other injuries may mimic TFCC tears on physical examination, most hand surgeons rely on wrist MDCT and/or MR arthrographic findings to direct clinical management $[3,7,14]$.

The TFCC consists of the articular disc (also known as the triangular fibrocartilage, TFC), the palmar and dorsal DRULs, the palmar and dorsal ulnocarpal (ulnolunate and ulnotriquetral) ligaments, the meniscus homologue, the ulnar collateral ligament, and the sheath of the ECU tendon $[10,15]$. The ulnar attachment of the TFCC is composed of two distinct laminae which are separated by the ligamentum subcruentum: the proximal lamina is oriented vertically and inserts on the ulnar fovea, whereas the distal lamina runs horizontally and inserts on the ulnar styloid process [16]. To our knowledge, dorsal peripheral tears of the TFCC, with or without associated ulnocarpal ligaments injury, have rarely been reported in the literature so far [4-6]. Our case seems to be the first to demonstrate partial separation of both palmar and dorsal DRULs from the articular disc, forming a bucket-handle tear of the TFCC (in analogy with a superior labral anteroposterior (SLAP) 3 lesion, Figs. $1 b-d$ and $2 c-d)$. The radiological profile of this complex peripheral lesion, which could be regarded as a combination of Palmer class 1B (ulnar attachment tear of TFCC distal lamina) and 1C (distal palmar and dorsal tears of the DRULs), was in the form of two discs connected by a fine thread and mimicked the appearance of a pair of sunglasses upon sagittal reformatted wrist MR arthrographic images (Fig. 2c). With the successful introduction of 3-Tesla MR imaging of the wrist, isotropic high-resolution MR images are now obtained in clinical routine. As the sensitivity, specificity, and accuracy of 1.5-T wrist MR imaging for traumatic peripheral tears (classes $1 \mathrm{~B}$ and $1 \mathrm{C}$ ) were previously reported to be as low as 17,79 , and $64 \%$, respectively [17], the analysis of high-resolution sagittal reformatted MR arthrographic images might be helpful in detecting more dorsal peripheral TFCC tears and their associated abnormalities, such as extrinsic carpal ligaments injuries. However, further studies are needed to assess the utility of those sagittal reformatted MR images.

Peripheral tears of the TFCC are important to recognize for several reasons. First, due to the rich blood supply of the peripheral portion of the TFCC, those tears portend good healing after open or arthroscopic surgical repair [4, 9, 18]. Second, those injuries may be associated with DRUJ and/or wrist instability. Indeed, Munk et al. evaluated the changes in wrist stability after experimental traumatic TFCC lesions [8]. They concluded that wrist stability was significantly related to Palmer class $1 \mathrm{C}$ lesions, in contrast to the other classes of traumatic TFCC injuries which mainly affected the stability of the DRUJ. Third, due to the loss of the cushion function of the TFCC for the ulnar carpus, peripheral tears may lead to degenerative changes in the radiocarpal joint and/or the DRUJ [10, 12].

Finally, we have to acknowledge two limitations of our case report. First, while we believe that the complexity of this lesion would only be fully appreciated with wrist MR arthrography, we have not had the opportunity to review the initial standard MR imaging study. Second, as the patient was rapidly lost to follow-up after surgery, we have not been able to evaluate the efficacy of surgical repair of this bucket-handle tear of the TFCC and its potential long-term complications.

Acknowledgments The authors gratefully thank Philippe Clavel for his computer graphics work.

Funding or grant None.

\section{References}

1. Palmer AK. Triangular fibrocartilage complex lesions: a classification. J Hand Surg Am. 1989;14:594-606.

2. Palmer AK. Triangular fibrocartilage disorders: injury patterns and treatment. Arthroscopy. 1990;6:125-32.

3. Potter HG, Asnis-Ernberg L, Weiland AJ, Hotchkiss RN, Peterson MG, McCormack Jr RR. The utility of highresolution magnetic resonance imaging in the evaluation of the triangular fibrocartilage complex of the wrist. J Bone Joint Surg Am. 1997;79:1675-84.

4. Estrella EP, Hung LK, Ho PC, Tse WL. Arthroscopic repair of triangular fibrocartilage complex tears. Arthroscopy. 2007; 23:729-37.

5. Machiels F, Moermans JP, Brutus JP. Arthrographic and CT arthrographic findings in dorsal peripheral detachment of the triangular fibrocartilaginous complex. JBR-BTR. 2001;84:114-7.

6. Morisawa Y, Nakamura T, Tazaki K. Dorsoradial avulsion of the triangular fibrocartilage complex with an avulsion fracture of the sigmoid notch of the radius. J Hand Surg Eur Vol. $2007 ; 32: 705-8$. 
7. Watanabe A, Souza F, Vezeridis PS, Blazar P, Yoshioka H. Ulnar-sided wrist pain. Part II: clinical imaging and treatment. Skeletal Radiol. 2010;39:837-57.

8. Munk B, Jensen SL, Olsen BS, Kroener K, Ersboell BK. Wrist stability after experimental traumatic triangular fibrocartilage complex lesions. J Hand Surg Am. 2005;30:43-9.

9. Corso SJ, Savoie FH, Geissler WB, Whipple TL, Jiminez W, Jenkins N. Arthroscopic repair of peripheral avulsions of the triangular fibrocartilage complex of the wrist: a multicenter study. Arthroscopy. 1997;13:78-84.

10. Vezeridis PS, Yoshioka H, Han R, Blazar P. Ulnar-sided wrist pain. Part I: anatomy and physical examination. Skeletal Radiol. 2010;39:733-45.

11. Raskin KB, Beldner S. Clinical examination of the distal ulna and surrounding structures. Hand Clin. 1998;14:177-90.

12. Sachar K. Ulnar-sided wrist pain: evaluation and treatment of triangular fibrocartilage complex tears, ulnocarpal impaction syndrome, and lunotriquetral ligament tears. J Hand Surg Am. 2008;33:1669-79.
13. Tay SC, Tomita K, Berger RA. The "ulnar fovea sign" for defining ulnar wrist pain: an analysis of sensitivity and specificity. J Hand Surg Am. 2007;32:438-44.

14. Moser T, Khoury V, Harris PG, Bureau NJ, Cardinal E, Dosch JC. MDCT arthrography or MR arthrography for imaging the wrist joint? Semin Musculoskelet Radiol. 2009;13:39-54.

15. Theumann NH, Pfirrmann CW, Antonio GE, Chung CB, Gilula LA, Trudell DJ, et al. Extrinsic carpal ligaments: normal MR arthrographic appearance in cadavers. Radiology. 2003;226:171-9.

16. Benjamin M, Evans EJ, Pemberton DJ. Histological studies on the triangular fibrocartilage complex of the wrist. J Anat. 1990;172:59-67.

17. Haims AH, Schweitzer ME, Morrison WB, et al. Limitations of MR imaging in the diagnosis of peripheral tears of the triangular fibrocartilage of the wrist. Am J Roentgenol. 2002;178:419-22.

18. Thiru RG, Ferlic DC, Clayton ML, McClure DC. Arterial anatomy of the triangular fibrocartilage of the wrist and its surgical significance. J Hand Surg Am. 1986;11:258-63. 